\title{
Transferencia del rol paterno a hijos (parentalización) en el Centro Poblado de Chala Nueva - San José de Quero
}

\author{
Transfer of the paternal role to children (parenting) in the Chala Nueva Populated \\ Center
}

Ana Manrique Canchari' (D) Luz María Macha Guerra² (D)

\begin{abstract}
Cómo citar
Manrique Canchari, A. y Macha Guerra, L. (2021). Transferencia del rol paterno a la infancia rural (niño, púber y adolescente) (parentalización) - Centro Poblado de Chala Nueva - San José de Quero. Socialium, 5(1), 196-206. https://doi.org/10.26490/uncp.sl.2021.5.1.630
\end{abstract}

${ }^{1}$ Magister en Políticas Sociales con mención en Promoción de la Infancia, Universidad Nacional del Centro del Perú, Huancayo,

Perú.

amanrique@uncp.edu.pe Google Scholar

2 Licenciada en Trabajo Social, Universidad Nacional del Centro del Perú, Huancayo, Perú.

Imacha@uncp.edu.pe Google Scholar

Arbitrado por pares ciegos Recibido: 09/10/2020 Aceptado: $23 / 11 / 20$

\section{RESUMEN}

Una característica del poblador andino asentado En la Provincia de Concepción, Región Junín, es el desarrollo de la actividad ganadera; en ella se involucran los integrantes de la familia rural conformando las empresas familiares. El objetivo de la investigación es identificar como se presenta la transferencia del rol paterno a la infancia rural (niño, púber y adolescente) (parentalización). La dinámica familiar es formativa, promueve el desarrollo de sus integrantes, así el infanto (niño, pre púber, púber y adolescentes) participan activamente en las actividades agropecuarias, los padres se desplazan a las chozas y transfieren el rol adulto al infanto, produciéndose la parentalización. Estudio cualitativo, de diseño fenomenológico, muestra las prácticas del adulto andino sobre los procesos de parentalización rural; se entrevistan a 7 infantos de ambos sexos con una guía de entrevista en profundidad. Los hallazgos muestran que progenitores no hacen uso de sus obligaciones y trasfieren inapropiadamente deberes a sus hijos de ambos sexos por espacios prolongados, cuando se desplazan a las chozas en correspondencia al calendario ganadero anual (apareamiento, nacimiento, beneficios y venta de animales ovinos y vacunos); el desplazamiento adulto a las ferias locales compromete seriamente los derechos y el necesario proceso evolutivo del niño, de estudiar, recrearse, jugar, etc. Se concluye que los padres dedicados a actividades ganaderas, transfieren a sus hijos, en su mayoria mujeres, obligaciones adultas como el cuidado de sus hermanos, asistencia a la escuela, cuidado de animales (empresa familiar) y de terceros, comprometiendo el desempeño escolar y vulneración de sus derechos.

Palabras clave: tranferencia; parentalización; roles; infanto.

\section{ABSTRACT}

A characteristic of the Andean settlers settled in the Concepción Province, Junín Region, is the development of livestock activity; the members of the rural family are involved in it, forming family businesses. The objective of the research is to identify how the transfer of the paternal role to rural childhood (child, puberty and adolescent) (parenting) is presented. Family dynamics is formative, it promotes the development of its members, thus the infant (child, pre-pubescent, puberty and adolescents) actively participate in agricultural activities, parents move to the huts and transfer the adult role to the infant, producing the parenting. Qualitative study, of phenomenological design, shows the practices of the Andean adult on the rural parenting processes; 7 infants of both sexes are interviewed with an in-depth interview guide. The results show that parents do not make use of their obligations and inappropriately transfer duties to their children of both sexes for long periods of time, when they move to the huts according to the annual livestock calendar (mating, birth, benefits and sale of sheep and cattle); adult travel to local fairs seriously compromises the rights and the necessary evolutionary process of the child, of studying, recreation, playing, etc. It is concluded that parents engaged in livestock activities, transfer to their children, mostly women, adult obligations such as caring for their siblings, school attendance, animal care (family business) and third parties, compromising school performance and violation of their rights.

Keywords: transfer; parenting; roles; infant. 


\section{Introducción}

El Provincia de Concepción (Junín), geográficamente cuenta con una zona altina con diversos pisos ecológicos, el Distrito de San José de Quero y el Centro Poblado de Chala Nueva se ubican en el espacio denominado "quechua"; los mismos que colindan con la Provincia de Chupaca en el acceso inicial y con el Distrito de Yauyos en la parte superior del piso ecológico, dicha configuración geográfica proporciona a toda esta zona como un espacio estratégico. Sus habitantes se dedican a las actividades agropecuarias y el comercio; el adulto varón y/o mujer, son dueños, rentan o son cuidadores de los predios destinados al desarrollo pecuario.

En correspondencia al calendario anual pecuario, los adultos en permanente dinamicidad se desplazan de entre el Centro Poblado de Chala Nueva a las ferias locales y a las zonas pecuarias; por tal motivo deben distribuir su tiempo para asistir al apareamiento, nacimiento, esquila, beneficio del ganado (venta de carne y viseras) y al comercio. En este proceso, los progenitores proporcionan el mayor interés a las actividades pecuarias y de comercio, descuidando el rol de padres, transfiriendo el papel a personas adultas mayores (abuelos) y a los niños, púberes y adolescentes; el adulto delega a grupos que biológicamente no están preparados para asumir tareas adultas. La delegación de funciones a través de tareas, cuestiona, amenaza, advierte al niño el cumplimiento de las obligaciones.

Boszormenyi y Spark (2003) plantean que la parentalización es la distorsión o la falta de las fronteras entre dos o más subsistemas de la familia, de tal manera que los niños asumen roles y responsabilidades que por lo general son reservadas para adultos, causando en ellos posibles trastornos psíquicos que pueden influenciar en sus relaciones personales y sociales. Minuchin (1982) menciona que la parentalización ocurre cuando el subsistema parental se apoya en un hijo para desempeñar ciertas funciones en la familia; es decir, el niño sacrifica sus propias necesidades de atención, comodidad y orientación con el fin de acomodar y cuidar las necesidades de los padres. La parentalización ha sido definida por diversos autores para referirse a problemas en la relación familiar que implican la inversión de roles funcionales y/o emocionales entre sus integrantes, donde, por ejemplo, los hijos llegan a desempeñarse como padres.

Rosado (2018) advierte que la parentalización es cuando los niños adoptan el rol de sus padres, aprecia además que "cuando los roles infantiles se invierten y el hijo se convierte en padre o madre, produce una confusión de identidad en la familia".

La transmisión inapropiada de obligaciones que le corresponde al adulto violenta los derechos del infanto. Según el Fondo de Población de las Naciones Unidas (2018), en el enfoque de derechos 
puntualiza que los derechos son iguales e inalienables para todos los seres humanos, estableciendo bases para la libertad, la justicia y la paz en el mundo, según la Declaración Universal de Derechos Humanos, adoptada por la Asamblea General de las Naciones Unidas en 1948, esta situación compromete biológica y emocionalmente al infanto, situándolos en condiciones de desarraigo de orfandad, alejándolos de la necesaria vinculación afectiva, Bowlby (1998) en la teoría del apego menciona que "una forma de conceptualizar la propensión de los seres humanos a formar vínculos afectivos fuertes con los demás es el apego, y de extender las diversas maneras de expresar emociones de angustia, depresión, enfado cuando son abandonados o viven una separación o pérdida".

El estudio pertenece a la línea de investigación: Desarrollo Humano y Políticas Sociales de la Facultad de Trabajo Social de la Universidad Nacional del Centro del Perú

\section{Métodos}

Estudio de naturaleza cualitativa, el nivel de investigación corresponde al estudio descriptivo, según Rivas (1992), la investigación descriptiva tiene como objetivo recabar información acerca del fenómeno para describir sus implicancias o hechos a partir de criterios. El estudio observa y describe a 7 infantos (niños, púberes y adolescentes) con experiencia rural involucrados en situaciones de parentalización, asumiendo roles adultos en el Centro Poblado de Chala Nueva del Distrito de San José de Quero - Concepción, 2019.

El tipo de investigación es básica; Muntané (2010) menciona que la investigación básica, también llamada pura, teórica o dogmática, se caracteriza porque se origina en un marco teórico y permanece en él, el objetivo es incrementar los conocimientos científicos, pero sin contrastarlos con ningún aspecto práctico; el estudio está orientado a observar e identificar para describir el comportamiento de niños, púberes y adolescentes que asumen inapropiadamente el rol que le corresponde al adulto varón y/o mujer (padres). Se observa al infanto en el espacio familiar, escolar.

Investigación cualitativa de diseño fenomenológico, Martinez (2001) plantea que la fenomenología es el estudio de los fenómenos tal como son vividos, experimentados y percibidos por el hombre; su énfasis primario está puesto en el fenómeno mismo, es decir, en lo que se presenta y revela a la propia conciencia y del modo como lo hace, con toda su creación y particularidad. Husserl (1988) sostiene que la fenomenología "es un método que intenta entender de forma inmediata el mundo del hombre mediante una visión intelectual basada en la intuición de la cosa misma, es decir, el conocimiento se adquiere válidamente a través de la intuición que conduce a los datos inmediatos y 
originarios". El diseño fenomenológico permite identificar, observar, entrevistar a los infantos en el espacio familiar y escolar rural; se selecciona intencionadamente a 7 infantos (niños, púberes y adolescentes) asentados en los espacios rurales familiares con escolaridad, a cargo de sus hermanos que estudian, participan en el apoyo del cuidado a sus hermanos menores (alimentación, vestido y asistencia al colegio).

Se les aborda con una guía de entrevista en profundidad y una guía de observación.

\section{Resultado}

La entrevista en profundidad permite visualizar las experiencias familiares de niños, púberes y adolescentes que asumen el rol adulto.

Los 7 entrevistados tienen hermanos:

E1: Sí, tengo 2 hermanos menores. E2: No, tengo hermanos mayores. E3: Sí, nosotros somos 5 hermanos y yo soy la segunda. E4: Sí, tengo una hermana menor. E5: Sí, tengo dos hermanos. E6: No ya no tengo, yo soy la menor. E7: Un hermano mayor por dos años

Sostienen que asumen el cuidado cuando sus padres no están en casa:

E1: A veces, cuando mis papás no están en mi casa. E2: no responde. E3: $\mathrm{Si}$, casi siempre porque mis papás trabajan y no hay con quien dejar a mis chiquitos. E4: A veces, cuando no están mis papás. E5: A veces, cuando mi mamá se va a la choza o a trabajar. E6: Cuando no está mi mamá yo preparo mis alimentos. E7: No lo cuido, pero si cocino cuando no está mi mamá

Horario, apoyan en las mañanas, tardes, noches:

E1: Por las mañanitas y por las tardes. Los días que mis papás no están que es más que nada cuando se van a vender a las ferias como los viernes y sábados. E2: no responde. E3: Los cuido en las mañanas, en las tardes y en las noches. Los días que mis papás están trabajando o están en la choza. E4: Solo cuando no están mis papás, mayormente sábados y domingos. E5: A veces mi mamá se va por 20 días, o por todo un día y ahí es cuando los cuido, no hay fechas fijas. E6: Cada que se va a trabajar. E7: Solo cocino, cuando mi mamá no está 
El tiempo del cuidado de los hermanos, se efectúa por días, uno, es más extenso cuando se desplaza a las chozas (...) sábados y domingos ..."

Escuela: Los púberes y adolescentes al cuidado de menores van a la escuela:

E1: Si voy al colegio. E2: Si. E3: Si. E4: Al colegio. E5: Si voy. E6: Al colegio. E7: Al colegio

Hacen sus tareas escolares:

E1: Si. E2: Si. E3: Si. E4: Si. E5: Si. E6: Si. E7: Si

Apoyo en las tareas escolares:

E1: Tengo que ayudar a mis hermanos las cosas que yo sé. E2: no responde. E3: Si, mis hermanitos no saben muchas cosas y como soy el mayor tengo que ayudarles. E4: Si a mi hermanita le ayudo sus tareas del jardín. E5: A mi hermanito le ayudo, mi hermana a veces me ayuda. E6: no responde. E7: No

Observamos que los púberes y adolescentes asisten al colegio, apoyan a sus hermanos en sus tareas escolares, evidenciándose una recargada labor.

Cuidado de animales: Apoyan en el cuidado de animales (gallinas, cuyes, vacas, carneros, cerdos, entre otros)

E1: Sí, tengo una vaca y gallinas. E2: Sí, tengo unos carneros y cuyes. E3: Sí, tengo una vaca, un chancho y cuyes. E4: Gallinas y cuyes. E5: Si, tengo gallinas y un pachito. E6: Un perro un gato y cuyes. E7: Tengo gallinas, dos perros y 4 gatos

El cuidado de sus hermanos, ir al colegio y ver a sus animales; es una ardua tarea para el infanto parentalizado comprometiendo el desempeño escolar.

E1: A veces no, porque cuando mi mamá no está tengo que cuidar a mis hermanos, a los animales. Me canso mucho. E2: Sí, porque mi abuelita me ayuda. E3: A veces se me hace difícil, tengo que atender a mis hermanos, a los animales, ya he llegado tarde varias veces. Pero que se hace tengo que ayudar en mi casa porque soy la mayor. E4: $\mathrm{Si}$, como son pequeños no hay mucho que hacer, cuando tienes vacas o carneros, hay es el problema. E5: Si. E6: Si. E7: Si

La entrevista en profundidad muestra que las adolescentes mujeres, asumen por ser la hermana mayor, tareas extenuantes. 
Concluimos, que existe delegación o transferencias de funciones adultas a adolescentes generalmente mujeres, es permanente; se efectúa de 2 a 3 días a la semana y es más prolongado cuando los padres se desplazan a las chozas. Es evidente la transferencia de roles adultos o parentalización rural.

\section{Discusión}

Las familias rurales en los espacios interandinos desarrollan prácticas de cuidado y atención familiar transmitidas de generación en generación; así los abuelos, tíos (familia extensa) transmite a la familia nuclear la incorporación de todos sus integrantes en las tareas para impulsar la empresa familiar. Se incorpora en su proceso a todos los miembros y se les encomienda tareas y obligaciones, según la edad.

Hipótesis especifica 1: "Los infantes rurales del Centro Poblado de Chala Nueva, del Distrito de San José de Quero durante el año 2019, asumen el cuidado de sus hermanos: preparación de alimentos, envío a la escuela, cuidado de animales menores y ganado".

El diseño fenomenológico muestra las experiencias producto de las entrevistas en profundidad y la observación; se observa las prácticas familiares, se observa la experiencia de la Entrevistada 1 en el ambiente familiar cuando sus padres se han desplazado a las chozas y/o ferias: "La adolescente prepara apresuradamente el desayuno, encarga a terceros el desplazamiento del ganado a los lugares de pastoreo, viste a sus hermanos para la asistencia a la escuela... la adolescente va corriendo al colegio y llega tarde"

Existe una asociación entre las prácticas familiares intergeneracionales y la transmisión inapropiada de roles; si bien el niño, púber y adolescente debe formarse para el futuro, asumiendo ciertas responsabilidades; en este contexto las pautas de crianza son formativas; sin embargo, cuando la parentalización equivoca los roles y transfiere obligaciones a los hijos se asiste a procesos de desnaturalización de los roles familiares. Minuchin (1982) en la Escuela Estructural, da cuenta de la existencia de subsistemas en la estructura familiar, definiendo una dinámica familiar que va a determinar la ejecución de roles, jerarquías, normas y límites. La participación de los infantos en labores que le corresponde al adulto "priva" a los niños de su niñez, su potencial y su dignidad que es perjudicial para el desarrollo físico e intelectual (Organización Internacional del Trabajo, 2019).

Aporta en la investigación Hernández (2016, párr. 5) cuando sostiene “...hay muchos casos donde los padres deben mantenerse ausentes o distantes tanto física como emocionalmente de sus hijos, ya 
sea por razones laborales o sociales... los hijos (generalmente el hijo mayor) empieza a asumir responsabilidades y tareas propias del subsistema parental...".

Se observa en el ambiente escolar a los informantes que llegan apresurados, desesperados, atolondrados, cuando la clase se ha iniciado.

El estudio se apoya en Sánchez (2020) cuando afirma que "existen tipos de parentificación física o instrumental... cuando se espera que los niños se hagan cargo de las necesidades domesticas o económicas, la preparación de las comidas, el cuidado de otros hermanos..."

La ayuda que ejerce el púber y/o adolescente se extiende al acompañamiento de sus hermanos a la escuela, comprometiendo el propio desempeño personal.

E6: Es difícil hacer todo... por eso llego tarde al colegio.

El asumir roles que no le corresponde, temerariamente compromete al cuidador... la depresión “... la persona acaba agotada (proceso de indefensión aprendida) al cuidar de los demás y no entender porque nadie lo cuida".

La investigación se sustenta en Rovetta (2016), cuando refiere que el termino de hijo parental se refiere a los niños que hacen la función de apoyo de la madre o padre, sustituyendo a la pareja y desempeñando el rol de padres de sus hermanos e incluso de sus padres, asumiendo las responsabilidades de un adulto. En algunos casos el hijo parental se encarga del cuidado de uno o ambos padres cuando estos son mayores; en otros, el hijo toma las responsabilidades de un adulto cuando uno o ambos padres son aún jóvenes. Por lo general encontramos este rol en el hijo del sexo opuesto al padre, pero también puede presentarse en el hijo del mismo sexo, de igual manera por lo general este papel suele tomarlo el hijo o hija mayor, aunque a veces recae en cualquier otro.

Se concluye: Los infantes rurales (niños, púberes y adolescentes) asumen roles que le corresponden a los adultos, el cuidado de sus hermanos, lo hacen porque sus padres tienen que trabajar y reconocen que deben ayudar, se acepta la hipótesis especifica 1.

Hipótesis general: Padres rurales del Centro Poblado de Chala Nueva - San José de Quero por actividades pecuarias (ganadería) se desplazan a las ferias, chozas por espacios prolongados; transfieren obligaciones a sus menores hijos (niño, púber y adolescente).

Un rasgo peculiar de los espacios altoandinos es la participación en actividades pecuarias (ganaderas), el ganado se encuentra en los espacios denominados chozas, alejados del Centro Poblado; la dinámica ganadera exige la permanencia de los cuidadores en dichas zonas; en estos periodos los adultos se desplazan a las chozas para resolver las exigencias del calendario pecuario o 
ganadero. Es en estos procesos que los adultos o progenitores delegan sus funciones parentales a sus hijos, persuadiéndoles u obligándoles a asumir las obligaciones porque ellos deben trabajar.

Parra (2015) refiere que la parentalización visibiliza parte de las formas de crianza de los hijos e hijas, además de las formas de relacionarse entre ellos y ellas. La primera a nivel general, resulta en un abandono, negligencia, escasa o nula afectividad y una asunción del rol paterno por parte del hijo mayor. Esta lógica de cuidado posiciona al hermano o hermana mayor como el eje rector de autoridad en la familia. Por ello, el sistema parental se ubica con las dos figuras paternas más un hijo parentalizado. Este último como víctima más visible de la vulneración de su niñez, ya que debe asumir las responsabilidades del cuidado de sus hermanos y hermanas menores, asumiéndose como adulto responsable. Además de ello, el trabajo de los padres es parte esencial para contextualizar estas formas de relaciones entre padres e hijos. Así, las largas jornadas de trabajo y la alta exigibilidad de ocupaciones no permiten a los trabajadores la asunción de sus derechos y responsabilidades como padres, siendo así también las padres víctimas de la estructura social que acarrea la pobreza.

E4: Tengo que apoyar, porque ellos deben trabajar... tenemos deudas.

Según Pankseep (2017), existen circuitos cerebrales que afectan o comprometen al ser humano es la pérdida del apego, el miedo, el temor o el deseo de que las cosas cambien. Los informantes refieren y reiteran que les gustaría que esta situación cambiara, que ellos puedan desempeñarse como cualquier adolescente y no asumieran responsabilidades de sus padres, les gustaría tener tiempo para sus obligaciones personales, para vivir el momento, dedicarse al juego, al deporte y otras actividades propias de su edad.

E6: Me gustaría que mis padres no salgan, se queden o nos cuiden... no es posible tienen que trabajar.

Bowlby (1998) refiere que la teoría del apego es una forma de conceptualizar la propensión de los seres humanos a formar vínculos afectivos fuertes con los demás y las diversas maneras de expresar emociones de angustia, depresión.

Para Domínguez, et al. (2017) es posible clasificar el fenómeno de parentalización como un tipo de negligencia emocional, ya que desde el momento en que se le otorga a un niño una tarea que excede sus capacidades físicas y/o emocionales, necesariamente el padre o cuidador está ignorando cuáles son las reales capacidades que el niño posee de acuerdo con su edad. Sin embargo, a diferencia de otros tipos de negligencia o maltrato, este fenómeno tiende ser invisibilizado puesto que es un medio para mantener el equilibrio familiar. Un ejemplo de lo anterior sucede cuando un padre, por motivos de trabajo, no tiene con quien dejar a sus hijos, por tanto, el hijo mayor queda a cargo de sus 
hermanos. En este caso el hijo cumple funciones que le corresponden al padre, obteniendo como beneficio el ser reconocido por su labor de "ayuda" para con sus padres o cuidadores, y estos últimos también se ven beneficiados por las tareas realizadas por su hijo.

Si bien las prácticas de crianza en el mundo rural son formativas, porque se prepara al adolescente para el futuro, ellos reconocen la importancia de implementar las empresas familiares, de generar responsabilidades, sin embargo, los padres descuidan aspectos importantes como la afectividad, la seguridad, el tiempo necesario en la escuela, vivir plenamente la adolescencia, participar con sus iguales en el juego, deporte, reuniones sociales; actividades necesarias para el proceso de formación. El asumir obligaciones adultas mella los derechos del infanto, se violentan los derechos consignados en la Convención del Niño y el Adolescente (UNICEF, 2006); se afecta o compromete el desarrollo integral del niño y adolescente, se violentan sus derechos.

El estudio se sustenta en Zamora (2013) cuando menciona que la parentalización es un fenómeno que afecta el ciclo vital de los niños, niñas y adolescentes, les afecta en su desarrollo psicoemocional, en la ubicación que ocupan en los subsistemas de la familia, pueden manifestar síntomas y transformarse en niños adultistas rígidos, con sentimientos de culpa y vergüenza si intentan desprenderse la parentalización. A pesar que falta aún políticas de conciliación trabajo y familia, se avanza en torno a la importancia de no tan sólo la satisfacción de necesidades básicas, si no al establecimiento de un vínculo afectivo entre padres e hijos, lo cual se logra con la participación activa de ambos progenitores, con la socialización de la equidad de género desde la infancia y con la protección de los derechos de la niños, niñas y adolescentes, asegurando su desarrollo integral y la concreción un proyecto de vida personal, desempeñando los padres adecuadamente sus roles, distribuyendo entre los adultos las labores de crianza y cuidado del hogar, no delegando excesivas responsabilidades en sus hijos(as), aunque si socializándolos paulatinamente de acuerdo a su edad y madurez para la vida como futuros padres.

Se concluye: Para el estudio, delegar responsabilidades tempranas a los adolescentes peligrosamente atentamos contra la integridad emocional afectiva, comprometiendo el ejercicio de los derechos, porque se afecta la necesaria libertad del ser humano, negándose la identidad, la dependencia, necesaria autonomía, el ejercicio de los derechos, se acepta la hipótesis general.

\section{Conclusión}

\section{Primera conclusión:}


En los espacios andinos, comprensión geográfica de la Provincia de Concepción, Distrito de San José de Quero, Centro Poblado de Chala Nueva, los padres dedicados principalmente a la actividad ganadera, transfieren a sus hijos (infanto, púberes y adolescentes), en mayoría mujeres, obligaciones adultas como el cuidado de sus hermanos, asistencia a la escuela, cuidado de animales (empresa familiar) y de terceros, comprometiendo el desempeño escolar y vulneración de sus derechos.

\section{Segunda conclusión:}

Según el calendario agropecuario, los adultos (padres) del Centro Poblado de Chala Nueva se desplazan a las chozas y las ferias locales por tiempos determinados; en este periodo delegan a sus menores hijos obligaciones o responsabilidades, comprometiendo el necesario desarrollo personal evolutivo, la libertad, recreación, juego; los hijos (as) parentales al asumir estas responsabilidades desarrollan cuadros emocionales (estrés) y carencias afectivas paterno - filial.

\section{Referencias}

Boszormenyi, I., y Spark, G. (2003). Lealtades Invisibles. Reciprocidad En Terapia Familiar Intergeneracional. Amorrortu Editores.

Bowlby, J. (1998). El apego. Paidós.

Domínguez, C., González, D., Navarrete, D., y Zicavo, N. (2017). Parentalización en familias monoparentales. Revista Ciencias Psicológicas, 13(2), 346-355. http://www.scielo.edu.uy/scielo.php?pid=S168842212019000200346\&script=sci_arttext\&tlng=pt

FondodePoblacióndelasNacionesUnidas. (2018). El enfoque basado en los derechos humanos. UNFPA. https://www.unfpa.org/es/el-enfoque-basado-en-los-derechoshumanos\#: : :text=Los\%20derechos\%20iguales\%20e\%20inalienables,las\%20Naciones\%20Un idas\%20en\%201948.

Hernández, G. (2016). Parentalización: cuando un NIÑO se convierte en PADRE. Psiquentelequia : https://psiquentelequia.com/parentalizacion-nino-padre/

Husserl, E. (1988). Las conferencias de París: Introducción a la fenomenología trascendental . Universidad Nacional Autónoma de México.

Martinez, M. (2001). Comportamiento humano: Nuevos métodos de investigación. Trillas.

Minuchin, S. (1982). Familias y Terapia Familiar. Celtia S.A.C.I.F.

Muntané, J. (2010). Introducción a la investigación básica. Rapd online, 33(3), 221-227. https://www.researchgate.net/profile/Jordi_Muntane2/publication/341343398_Introducci on_a_la_Investigacion_basica/links/5ebb9e7d92851c11a8650cf9/Introduccion-a-laInvestigacion-basica.pdf 
Organización Internacional del Trabajo. (2019). Trabajo infantil: qué es, causas y consecuencias. UNHCR ACNUR. https://eacnur.org/blog/trabajo-infantil-que-estc_alt45664n_o_pstn_o_pst/\#: :text=La\%200IT\%20explica\%20que\%20se,su\%20desarrollo \%20f\%C3\%ADsico\%20y\%20psicol\%C3\%B3gico.

Pankseep, J. (2017). Las emociones vistas por el psicoanálisis y la neurociencia: un ejercicio de conciliación. Revista Internacional de Psicoanálisis, (7). https://www.aperturas.org/articulo.php?articulo=155

Parra, A. (2015). Las Características de la Parentalización con Famílias en condiciones de Ausencia Parental por situaciones Laborables pertenecientes al Barrio Umbría [Tesis pregrado, Universidad Politécnica Salesiana Quito]. Repositorio institucional UPS. https://dspace.ups.edu.ec/bitstream/123456789/9707/1/UPS-QT07498.pdf

Rivas, J. (1992). Técnicas de Documentación Investigación I. Universidad Nacional Abierta.

Rosado, R. (2018). Parentalización, o cuando los niños adoptan el rol de sus padres. Psicología desde el alma. https://psicologiadesdeelalma.wordpress.com/2018/09/13/parentalizacion-ocuando-los-ninos-adoptan-el-rol-de-suspadres/\#: :text=Cuando\%20los\%20roles\%20infantiles\%20se,conoce\%20como\%20parentali zaci\%C3\%B3n\%20o\%20parentificaci\%C3\%B3n.

Rovetta, A. (2016). El hijo parental... una carga muy pesada. Psicología Integral Uruguay. https://www.psicologiaintegraluruguay.com/2016/10/el-hijo-parental-una-carga-muypesada.html\#: :text=una\%20carga\%20muy\%20pesada,octubre\%2011\%2C\%202016\&text=El\%20termino\%20de\%20hijo\%20parental,las\%20respon sabilidades\%20de\%20un\%20adulto.

Sánchez, G. (2020). Parentificación: niños que ejercen el rol de padres. La mente es maravillosa. https://lamenteesmaravillosa.com/parentificacion-ninos-que-ejercen-el-rol-de-padres/

UNICEF. (2006). Convención sobre los derechos del niño [Archivo PDF]. UNICEF. https://www.un.org/es/events/childrenday/pdf/derechos.pdf

Zamora, P. (2013). Fenómeno del hijo parentalizado en niños, niñas y adolescentes en contexto de vulneración de derechos [Monografía científica posgrado, Universidad del Bio-Bio]. Repositorio institucional UBIOBIO. http://repobib.ubiobio.cl/jspui/handle/123456789/506

\section{Contribución de las autoras}

AMC: Conceptualización, investigación, metodología, redacción: revisión y edición.

LMMG: Conceptualización, investigación, metodología, redacción: borrador original.

\section{Fuentes de financiamiento}

El estudio fue financiando por las autoras

\section{Conflictos de interés}

Las autoras no presentaron conflictos de interés durante el desarrollo de la investigación

\section{Correspondencia}

amanrique@uncp.edu.pe 